Boletín de la Sociedad Zoológica del Uruguay, 2020

Vol. 29 (2): 116-125

ISSN 2393-6940

https://journal.szu.org.uy

\title{
MACROINVERTEBRADOS BENTÓNICOS INDICAN EMPEORAMIENTO DE LA CALIDAD DE AGUA EN UNA ZONA DE INFLUENCIA URBANA EN EL RÍO NEGRO (URUGUAY)
}

\author{
Manuel Castro, Leandro Capurro, Guillermo Chalar \& Rafael Arocena \\ Sección Limnología. Facultad de Ciencias, Universidad de la República. Iguá 4225, Montevideo 11400, \\ Uruguay
}

Autor para correspondencia: mcastro@fcien.edu.uy

\section{RESUMEN}

Los sistemas fluviales están sometidos a una fuerte presión antropogénica que afecta su calidad de agua y biodiversidad. En Uruguay, el Río Negro es el mayor río interior y lo atraviesa de Este a Oeste luego de nacer en Brasil. Recibe los efluentes de algunas ciudades como Paso de los Toros y drena una cuenca con usos agrícola, ganadero y forestal. La Dirección Nacional de Medio Ambiente realiza un monitoreo de la calidad de agua del Río Negro desde el año 2009 con resultados que indican una calidad aceptable en todo el curso (excepto por el fósforo). Sin embargo, el río recibe de sus tributarios de menor orden principalmente materia orgánica, y efluentes domésticos de las ciudades ubicadas sobre los cursos principales. El presente estudio busca evaluar el impacto urbano y la capacidad de autodepuración del río comparando la comunidad de macroinvertebrados bentónicos entre zonas aguas arriba (control) y abajo de la ciudad de Paso de los Toros (contaminación y autodepuración).

Palabras Clave: Contaminación, Bioindicadores, Impacto Antrópico

\section{ABSTRACT}

Benthic macroinvertebrates indicate loss of water quality in a urban influenced area of Río Negro (Uruguay). River systems are subject to strong anthropogenic pressure that affects their water quality and biodiversity. The Río Negro is the largest inland river in Uruguay. It crosses the country from east to west after being born in Brazil. It receives the effluents from some cities such as Paso de los Toros and it drains a basin with agricultural, livestock and forestry uses. The National Environment Directorate has been monitoring the water quality of the Río Negro since 2009 with results that indicate an acceptable quality throughout the course (except for phosphorus). Nevertheless, the river captures from their small tributaries, mainly organic matter and domestic effluents from the cities located on the main courses. The present study seeks to assess the urban impact and the self-purification capacity of the river by comparing the benthic macroinvertebrates communities upstream (control) and downstream Paso de Los Toros city (pollution and self-purification) zones.

Key Words: Pollution, Bioindicators, Anthropic impact

\section{INTRODUCCIÓN}

A nivel mundial los sistemas fluviales están sometidos a una fuerte presión antropogénica que provoca cambios en la calidad del agua y en su biodiversidad. Estos cambios son consecuencia de los contaminantes provocada por actividades industriales, los asentamientos humanos, los plantíos y los campos ganaderos (Flint \& Davis, 2007; Salcedo et al., 2013). A pesar de lo anterior, los sistemas fluviales poseen la capacidad de autodepurarse, permitiéndoles recuperar calidad ecológica y biodiversidad (Sabater et al., 2009). Esta capacidad depende de tres aspectos: el caudal, que permitirá diluir el vertido; la turbulencia del agua, que aportará oxígeno al medio, favoreciendo la actividad microbiana y facilitando su degradación y de la naturaleza y volumen del vertido en relación al del curso receptor (Montes et al., 2013). Los componentes orgánicos en general se degradan por dilución, sedimentación, asimilación y descomposición (Valdés-Abellán et al., 2013). Sin embargo, los contaminantes químicos (detergentes, pesticidas, hidrocarburos, metales pesados) pueden persistir más tiempo en el ambiente e interrumpir los procesos de autodepuración del río (Arocena, 1996; Parra \& Rojas, 2003; Arocena et al., 2011).

Una manera de evaluar las zonas de contaminación y autodepuración en un río es mediante el monitoreo de sus organismos bentónicos. El uso de las comunidades bentónicas en los ríos como indicadores tiene numerosas ventajas debido a que las mismas se ven afectadas simultáneamente por múltiples factores de diversa naturaleza (Hellawell, 1986; Reynoldson, 


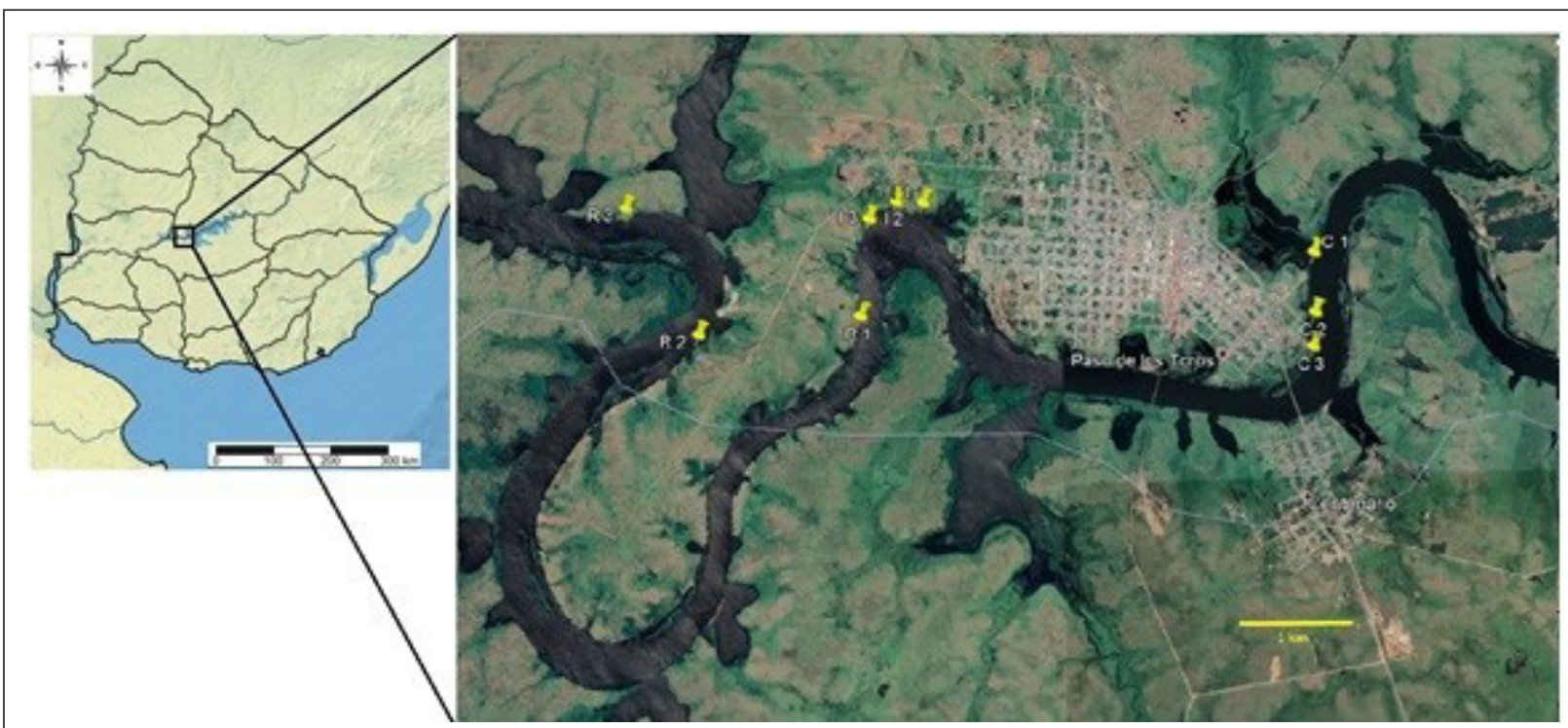

Fig. 1. Mapa de la zona de influencia de Paso de los Toros y las tres zonas y nueve sitios de muestreo: Control (C1, C2, C3), Impacto (11, I2, I3), Recuperación (R1, R2, R3).

1987; Plafkin et al., 1989; Rosenberg \& Resh, 1993), y dada su exposición permanente a las fuentes de contaminación, brindan una visión integrada de los diversos efectos y lo hacen a mediano y largo plazo. Existen índices bióticos que utilizan las abundancias de macroinvertebrados para determinar de una forma estándar la calidad del agua, sin embargo, el uso de índices para grandes ríos no está tan desarrollado como para otros sistemas acuáticos (Blocksom \& Johnson, 2009) y mucho menos en Sudamérica (Pave \& Marchese, 2005; Murphy et al., 2015).

El Río Negro es el curso de agua interior más importante del Uruguay, siendo relevante tanto por su longitud como por los usos actuales y potenciales de su cuenca, la más extensa dentro del país con 68.400 $\mathrm{km}^{2}$ (MVOTMA-DINAMA, 2011). La mayoría de los estudios de calidad de agua en el Río Negro comienzan en los años 90 y se centran en calidad de agua (Conde et al., 2002), ictiofauna (Amestoy, 2001), bivalvos invasores (Brugnoli et al., 2011) y cianobacterias (Gonzalez-Piana et al., 2017) en sus tres embalses. La Dirección Nacional de Medio Ambiente (DINAMA) realiza un monitoreo de la calidad de agua de todo el Río Negro desde el año 2009. Todos los parámetros medidos indican una calidad aceptable en todo el curso, excepto el fósforo total (80 a $90 \mu \mathrm{g} / \mathrm{L}$, MVOTMA-DINAMA, 2017).

El río capta los contaminantes principalmente orgánicos de los tributarios de menor orden, que drenan una cuenca con diferentes usos del suelo como ganadería, forestación, cultivos de secano y arroz
(MVOTMA, 2009). El curso principal es utilizado con distintos fines comerciales e industriales como la generación hidroeléctrica, riego, extracción de áridos, turismo y piscicultura, siendo además receptor de los efluentes de ciudades (DINAMA, 2011). La ciudad de Paso de los Toros se encuentra situada en la zona suroeste del departamento de Tacuarembó y sobre el margen derecho del Río Negro (Fig. 1). Cuenta con servicio de saneamiento y planta de tratamiento de aguas residuales, ubicada a metros de la orilla del Río Negro, al igual que un vertedero municipal de residuos sólidos.

El presente estudio tiene como objetivo evaluar el impacto urbano y la capacidad de autodepuración del río comparando la comunidad de macroinvertebrados bentónicos entre zonas aguas arriba (control) y abajo de la ciudad (Impacto y autodepuración).

\section{MATERIALES Y MÉTODOS}

Este estudio se realizó en tres zonas de un tramo de $17 \mathrm{~km}$ de largo sobre la orilla derecha (norte) del Río Negro, en los alrededores de la ciudad de Paso de los Toros, departamento Tacuarembó (-32.8181, 56.5064). Las zonas fueron nombradas como "Control" previa a la ciudad, "Impacto" ubicada al final de la zona urbana, y "Recuperación" aguas abajo de la ciudad (Fig. 1). En cada zona se ubicaron 3 sitios de muestreo para abarcar la posible heterogeneidad de hábitats presentes. 

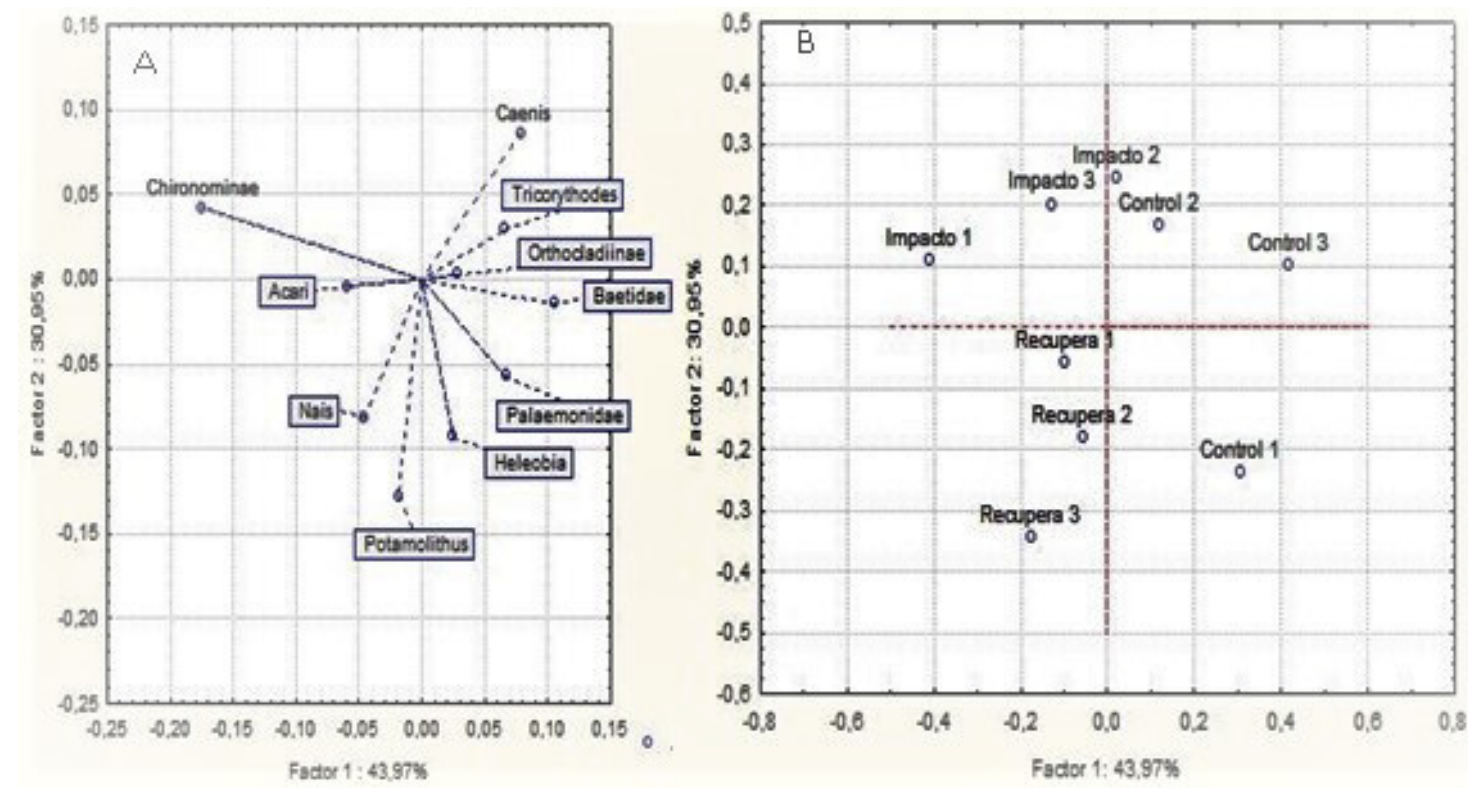

Fig. 2. Análisis de componente principal de las taxa más abundantes. A- contribución de las diferentes taxa a cada componente. B- nivel de correlación de los sitios con los factores principales.

La zona "Control" se extiende por aproximadamente $1 \mathrm{~km}$ desde la desembocadura del arroyo Sarandí, ubicado al Este de la ciudad, hasta una zona de camping y recreación, se encuentra $18 \mathrm{~km}$ aguas debajo de la represa hidroeléctrica Rincón del Bonete. La zona "Impacto" se extiende por 700 m, en zonas suburbanas aguas abajo de la ciudad y de su planta de tratamiento de aguas residuales. La zona "Recuperación" se extiende aguas abajo y por aproximadamente $10 \mathrm{Km}$, a lo largo de los cuales no se observan fuentes importantes de contaminación puntual al río (Fig.1).

El muestreo se realizó en junio del 2019, cuidando que el caudal proveniente del embalse Rincón del Bonete estuviera por debajo de $300 \mathrm{~m}^{3} / \mathrm{s}$ para evitar los efectos de deriva de los altos flujos de agua. Se midió in-situ temperatura, $\mathrm{pH}$, oxígeno, conductividad y turbiedad con sonda Horiba U51. Los artrópodos bentónicos fueron recolectados con una red $\mathrm{D}$ con entramado de malla de $500 \mu \mathrm{m}$ durante 2 minutos, usando la metodología descripta en Arocena et al. (2018). En cada sitio las muestras se tomaron por triplicado, abarcando distintos microhábitats de vegetación y sedimento. Cada muestra se depositó en un recipiente y fijó con etanol $70 \%$ (Merrit \& Cummins, 1996). Los organismos se separaron bajo microscopio estereoscópico y se identificaron al menor nivel taxonómico posible siguiendo claves locales y regionales (Brinkhurst \& Marchese, 1992, Lopretto \& Tell, 1995, Trivinho-Strixino \& Strixino ,1995, y Fernández \& Domínguez, 2001).
Se realizaron matrices con las principales métricas de los organismos y con los datos de los parámetros fisicoquímicos. Se calcularon, tomándose como indicadores, los parámetros comunitarios de abundancia relativa, riqueza, diversidad de Shannon $(\mathrm{H})$ y dominancia de Simpson (D), con el software libre PAST 3.0 (Hammer et al., 2001). En el mismo programa se realizaron análisis de similitud (ANOSIM) entre zonas y se determinó el porcentaje de disimilitud por análisis de SIMPER y las diferencias se analizaron en un Análisis de Escalamiento Multidimensional no paramétrico (nm-MDS), todos los análisis anteriores utilizaron distancias de Bray-Curtys.

En el programa STATISTICA 8.0 se determinó normalidad de datos mediante el test de ShapiroWilks y homogeneidad de varianza según el Prueba $C$ de Cochran, sin embargo, no todas las variables (biológicas y físico-químicas) cumplieron las dos condiciones, por lo que se prosiguió a transformar los datos en base a Reavie et al., (2010). A los datos se les aplico un análisis de varianza (ANOVA) y se prosiguió con un test de Turkey con aquellos que dieron resultados significativos (pd $\leq 0,05)$ Se tomaron los organismos cuya abundancia representó al menos un $1 \%$ del total de organismos hallados (César et al., 2019) un total de 10 taxa y se realizó un ANOVA para determinar diferencias entre las zonas y un test de Turkey para determinar cuáles taxa eran los que diferían. En el mismo programa, se realizó una correlación de Kendall-Tau entre las diferentes taxa, 
Tabla 1. Parámetros físico-químicos y geolocalización de los sitios de muestreo. Temp $=$ Temperatura $\left({ }^{\circ} \mathrm{C}\right)$, Conduc $=$ Conductividad $\left(\mu \mathrm{S} . \mathrm{cm}^{-1}\right)$.

\begin{tabular}{|c|c|c|c|c|c|c|}
\hline Estaciones & Coordenadas & Temp & $\mathrm{pH}$ & $0_{2} \%$ & Conduc. $\mu S . \mathrm{cm}^{-1}$ & Turbiedad (NTU) \\
\hline Control 1 & $-32.810892^{\circ},-56.497568^{\circ}$ & 13,9 & 8,0 & 106 & 75 & 1,8 \\
\hline Control 2 & $-32.814338^{\circ},-56.498057^{\circ}$ & 14,7 & 7,5 & 92 & 76 & 2,2 \\
\hline Control 3 & $-32.818338^{\circ},-56.498317^{0}$ & 13,8 & 8,1 & 102 & 110 & 2,6 \\
\hline Impacto 1 & $-32.805519^{\circ},-56.536142^{\circ}$ & 14,3 & 8,4 & 133 & 75 & 2,5 \\
\hline Impacto 2 & $-32.805053^{\circ},-56.539033^{\circ}$ & 13,9 & 8,7 & 131 & 77 & 2,3 \\
\hline Impacto 3 & $-32.808216^{0},-56.542148^{\circ}$ & 13,0 & 8,2 & 114 & 76 & 2,6 \\
\hline Recuperación 1 & $-32.816049^{\circ},-56.542494^{\circ}$ & 13,0 & 8,2 & 107 & 50 & 2,5 \\
\hline Recuperación 2 & $-32.817191^{\circ},-56.558552^{\circ}$ & 13,6 & 8,5 & 132 & 71 & 2,6 \\
\hline Recuperación 3 & $-32.806017^{\circ},-56.566277^{0}$ & 11,9 & 7,8 & 117 & 88 & 4,0 \\
\hline
\end{tabular}

los parámetros físico-químicos, los indicadores de calidad de agua y parámetros comunitarios con el fin de dilucidar las medidas de dependencia existente entre las variables utilizadas. Finalmente se hizo un Análisis de Componente Principal (ACP) en base al nivel de covarianza de los taxa principales, para caracterizar los sitios según los organismos.

Se aplicaron varios indicadores de calidad del agua a nivel de familia y sub-familia como el IBF-Chile y el BMWP-Colombia (Figueroa et al., 2003; Roldán \& Restrepo, 2008) y porcentaje de Chironomidae (\%Chiro). Se calculó el porcentaje de taxones considerados tolerantes (\%spp. tol) a la contaminación en cada sitio en base a las conclusiones obtenidas por Arocena et al. (2018) de los macroinvertebrados de arroyos vadeables de la cuenca del mismo río.

\section{RESULTADOS}

La conductividad varió entre 50 y $110 \mu \mathrm{S} / \mathrm{L}$, el pH entre 7.5 y 8.6 , la saturación de $\mathrm{O}_{2}$ entre 92 y $133 \%$, la temperatura entre 12 y $15^{\circ} \mathrm{C}$ y la turbidez entre 1.8 y 4.0 NTU. Solo el porcentaje de oxígeno tuvo diferencias significativas entre la zona Control e Impacto siendo mayor en ésta $(p<0,05)$ (Tabla 1$)$.

Se hallaron 5180 individuos, 40 familias de al menos 65 géneros entre todos los sitios, la lista de taxa hallados se muestran en la Tabla 2 . El taxón más abundante fue la sub-familia Chironominae, representando el $39 \%$ del total de los organismos hallados, seguido por Tricorythodes spp. (19\%), Caenis spp. (11\%) y Pothamolithus spp. (6\%). Los organismos recolectados y sus abundancias relativas se presentan en la Tabla 3.

Solo 10 taxa tuvieron una abundancia total mayor al $1 \%$ del total de organismos, y representan en conjunto el $92 \%$ de los macroinvertebrados recolectados (Nais spp., Heleobia spp., Potamolithus spp., Palaemonidae, Acari, Caenis spp., Baetidae, Tricorythodes spp., Chironominae y Orthocladiinae). Las familias Baetidae y Orthocladiinae mostraron correlaciones negativas con el porcentaje de oxígeno $(p<0, .05)$. El pH se correlacionó negativamente con Orthocladiinae. La diversidad de Shannon se correlacionó positivamente y la Dominancia negativamente con Palaemonidae y Baetidae, adicionalmente mostraron el patrón opuesto con Chironominae $(p<0.01)$. Las variables que dieron resultados significativos se presentan en la tabla 3.

\section{Sitios Control, Impacto, Recuperación}

Se hallaron diferencias significativas entre las comunidades bentónicas de los distintos sitios Control, Impacto y Recuperación mediante el ANOSIM ( $p=0.01$ y $R=0.33$ ). El análisis nm-MDS (estrés $=0.11$ ) muestra 3 grupos separados: Control, Impacto y Recuperación.

EI análisis SIMPER indicó que Control es más diferente de Impacto (50\% de disimilitud) que de Recuperación (49\%), que estas dos entre sí (40\%). Chironominae fue el taxón que más aportó a la disimilitud entre todas las zonas (34\%, 35\% y 19\% respectivamente). Se observaron diferencias significativas $(p<0.05)$ entre las abundancias relativas de Nais spp. (Recuperación > Control e Impacto), Palaemonidae (Control > Impacto) y Baetidae (Control $>$ Impacto).

No se encontraron diferencias significativas de la diversidad de Shannon entre las zonas, pero se observó la mayor diversidad en el sitio $\mathrm{C} 1\left(\mathrm{H}^{\prime}=2.4\right)$ y la menor diversidad en $\mathrm{I1}\left(\mathrm{H}^{\prime}=1.25\right)$. El índice de 
Tabla 2. Lista de taxa hallados en el presente estudio.

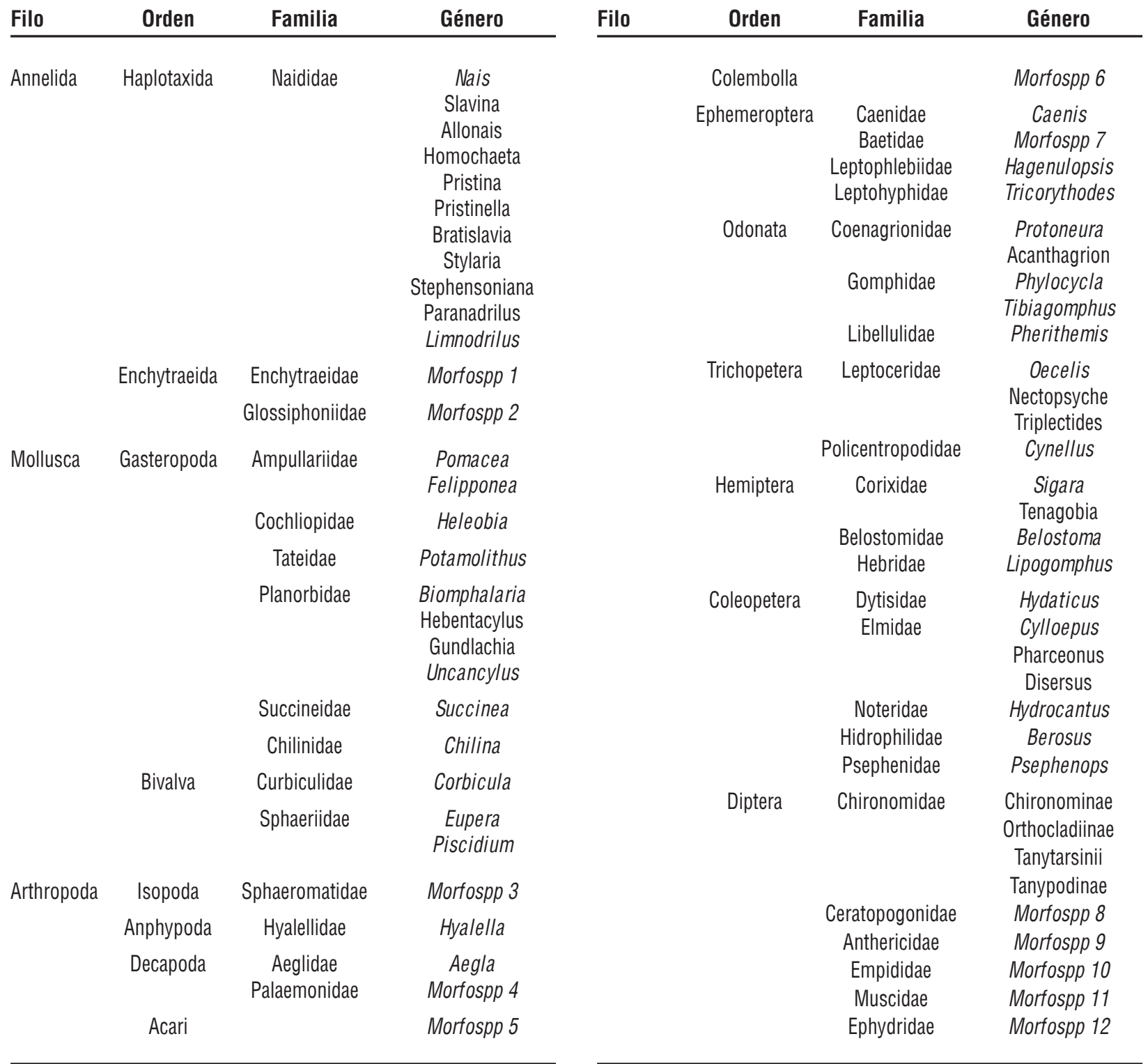

dominancia fue mayor en 11 (0.5) que en C1 (0.1).

De todos los índices de calidad de agua aplicados, los únicos que mostraron diferencias significativas entre zonas fueron \% Chiro y \% spp. tol, ambos mayores en Impacto que en Control. El BMWPColombia, el IBF-Chile, \% Chiro y \% spp. tol señalan a C1 como el sitio con mejor calidad de agua. Adicionalmente, el IBF, \% Chiro y \% spp. tol señalan a 11 como el sitio más contaminado, mientras que BMWP señala a 12, seguido por 11, como los más contaminados. En la Tabla 4 se muestran los valores de los índices utilizados y la interpretación del BMWP y el IBF.

\section{Análisis de componente principales}

El primer eje del ACP explica el $44 \%$ de la varianza total y el segundo el $31 \%$ (Fig. 2A). El primer factor se correlacionó positivamente con Baetidae (nivel de correlación $=0.96)$, Palaemonidae (0.67), Tricorythodes spp. (0.57), Caenis spp. (0.53) y Orthocladiinae (0.51), y negativamente con Chironominae $(-0.96)$ y Acari (0.96). El segundo factor se correlacionó positivamente con Caenis spp. (0.63) y negativamente con Potamolithus spp. (-0.90), Heleobia spp. (-0.79), Nais spp. (-0.67) y Palaemonidae (-0.57). Todos los sitios de Recuperación se correlacionan negativamente con ambos factores. A su vez Impacto se correlacionó 
Tabla 3. Nivel de correlación de Kendall-Tau de las diferentes variables estudiadas.

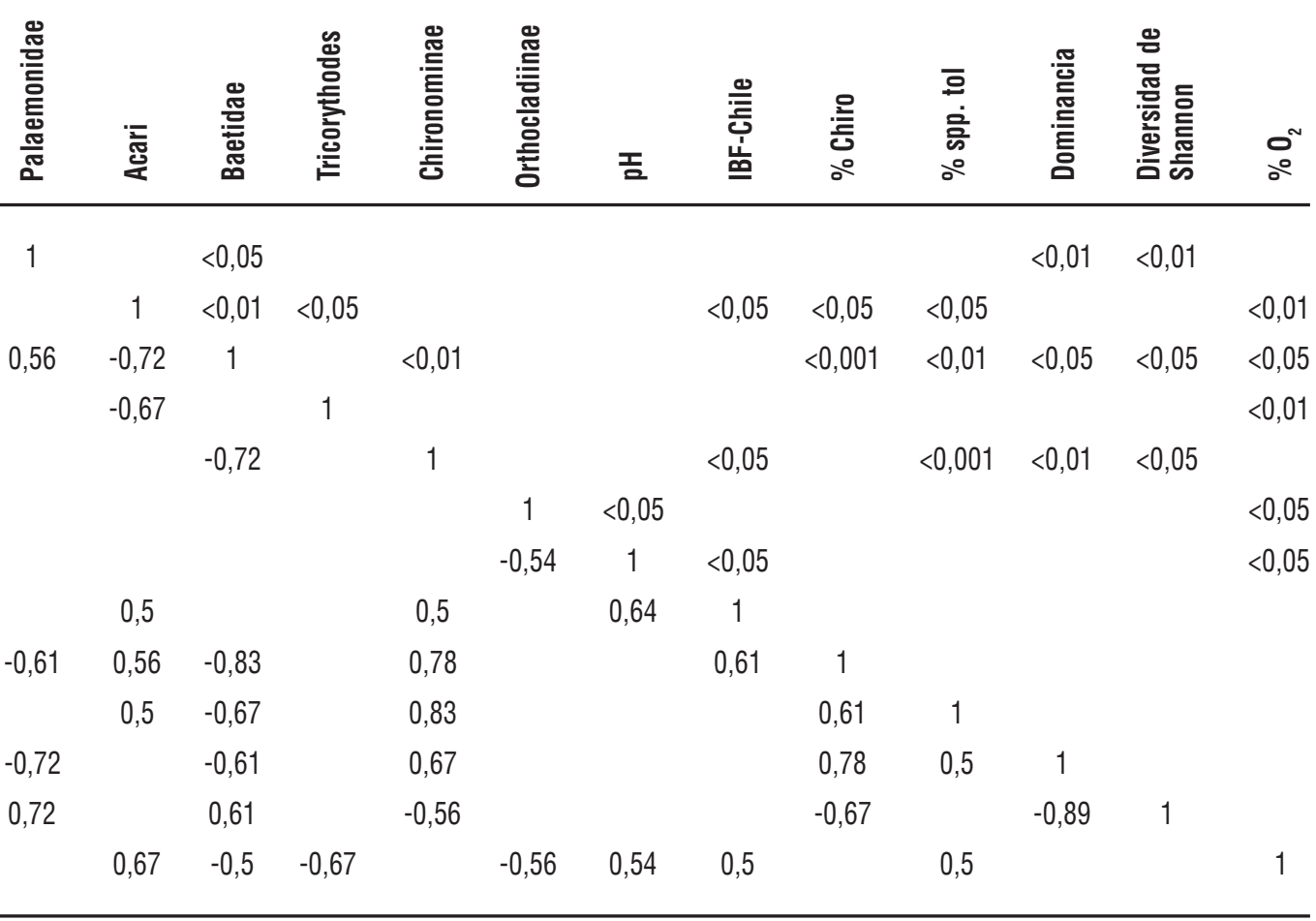

positivamente con el segundo factor, al igual que $\mathrm{C} 2 \mathrm{y}$ $\mathrm{C} 3$, y todo Control se correlacionó positivamente con el factor 1. (Fig. 2B).

\section{DISCUSIÓN}

Todos los sitios de Control se asociaron positivamente con el primer factor del ACP, el cual está correlacionado fuertemente a otro indicador de buena calidad del agua como es el género Tricorythodes (Vergara-Olaya, 2009; Chalar et al., 2011). La zona de Recuperación se diferencia de las otras por la mayor presencia del oligoqueto Nais spp. y por su correlación negativa con los dos primeros factores del ACP (Fig. 2B). Además, aunque no exista diferencias significativas en los promedios, se observa que los valores de la mayoría de los índices (\% Chiro y \% spp. tol, IBF, BMWP) indican mejor calidad de agua en Recuperación que en Impacto.

De los parámetros físico químicos solo el $\% \mathrm{O}_{2}$ fue significativamente mayor en la zona Impacto que en Control. Tales diferencias pudieron deberse a las distintas horas en que fueron muestreadas, ya que al progresar el día aumenta el porcentaje de saturación del oxígeno debido a la fotosíntesis favorecida por mayor disponibilidad de luz (Arocena \& Conde, 1999). Todos los puntos poseen $\mathrm{pH}$ y conductividad normales, excepto la conductividad en C3 que sería propia de ambientes más comprometidos, tal vez por su enclave en la zona de camping. La falta de diferencias en los parámetros físico-químicos del agua era esperable debido a que el tramo del río seleccionado no fue lo suficientemente largo y el caudal homogeneiza el ambiente. Si bien no existen grandes industrias que puedan aportar contaminación al río, la ciudad es una fuente puntual de contaminación, especialmente en épocas de lluvia, cuando la escorrentía arrastra materia orgánica, hidrocarburos, metales y otros contaminantes de las calles (Lee \& Bang, 2000; Flint \& Davis, 2007).

Según el nm-MDS I3 se encuentra más similar a R1 que a los otros sitios de Impacto. Esto podría evidenciar un comienzo del proceso de autodepuración del agua (Arocena, 1996), siendo que a partir de I1 todos los índices de calidad, excepto el BMWP, muestran una mejora. EI SIMPER indicó que Recuperación e Impacto se parecen más entre sí que a Control, lo cual es concordante con lo observado anteriormente

Las altas abundancias de Chironomidae se podría deber a que estos organismos predominan en ambientes que presentan disturbios frecuentes (Munn \& Brusven, 1991; Moog, 1993). Varios estudios, inclusive en la misma cuenca (Arocena et al., 2018), demuestran que aún la familia Chironomidae es un buen bioindicador de mala calidad del agua, al ser dominantes en ambientes contaminados y con estrés por metales pesados como es de esperar que suceda 
Tabla 4: Índices de calidad de agua utilizados y su interpretación. \% Chiro= abundancia relativa de Chironomidae. \% spp. tol = abundancia relativa de especies consideradas tolerantes a la contaminación.

\begin{tabular}{|c|c|c|c|c|c|c|}
\hline Sitio & $\%$ Chiro & \%spp. tol & & BMWP Colombia & & IBF-Chile \\
\hline Control 1 & 15,71 & 16,79 & 110,00 & Limpias & 3,44 & Excelente \\
\hline Control 2 & 35,77 & 35,12 & 85,00 & Contaminación ligera & 4,15 & Muy buena \\
\hline Control 3 & 21,72 & 16,74 & 67,00 & Contaminación ligera & 4,64 & Buena \\
\hline Impacto 1 & 71,07 & 70,32 & 74,00 & Contaminación ligera & 6,31 & Relativamente mala \\
\hline Impacto 2 & 44,16 & 35,06 & 53,00 & Contaminación moderada & 5,50 & Regular \\
\hline Impacto 3 & 52,70 & 53,82 & 74,00 & Contaminación ligera & 4,66 & Buena \\
\hline Recupera 1 & 43,63 & 41,94 & 91,00 & Contaminación ligera & 5,51 & Regular \\
\hline Recupera 2 & 40,17 & 42,34 & 87,00 & Contaminación ligera & 5,18 & Regular \\
\hline Recupera 3 & 42,52 & 41,89 & 90,00 & Contaminación ligera & 4,28 & Buena \\
\hline
\end{tabular}

a continuación de una ciudad (Roldán \& Restrepo, 2008; Figueroa et al., 2003; Zilli \& Gagneten, 2005; Baptista et al., 2007;). Se observa que hay mayores abundancias relativas en las zonas de Impacto y Recuperación que en Control, lo que podría deberse a la mejor calidad de agua en Control que en Impacto y Recuperación. Por lo anterior \%Chiro sería un indicador útil para determinar el impacto que están sufriendo los diferentes sitios, siendo que el punto con mayor valor de este índice es I1 que, por su cercanía a la salida del saneamiento, es el sitio más vulnerable a los efectos de la contaminación urbana. Contrariamente, C1 posee el menor valor de \%Chiro y es el punto menos proclive a recibir la contaminación de la ciudad. Los otros índices bióticos se comportaron de manera similar. La diversidad de Shannon puede poseer más influencia del tipo de hábitat que del nivel de contaminación. Según los valores de dominancia, C1, C3 y R2 serían ambientes con muy buena calidad del agua $(D<0.2)$ y ninguna estación sería mala $(D>0.6)$ (Vergara-Olaya, 2009).

El \%spp tolerantes diferenció Control de Impacto, este índice se correlacionó negativamente con Baetidae, una familia asociada a buena calidad del agua (Roldán \& Restrepo, 2008; Ambrioso, 2014) y positivamente con Acari, asociado a mala calidad del agua (Vergara-Olaya, 2009; Ambrioso, 2014).

La zona Control agrupa a varios taxa asociados a buena calidad de agua según el ACP. El BMWP y el IBF indican que más cerca de Paso de los Toros existe peor calidad del agua. Esto podría deberse al efecto de las actividades recreativas que se realizan en los márgenes del río. Sin embargo, la calidad del agua sigue teniendo categoría "buena" según los índices biológicos nombrados.

La zona de Impacto se caracteriza por poseer mayor cantidad de indicadores de mala calidad del agua, lo que concuerda con su ubicación geográfica aguas debajo de la ciudad y su planta de tratamiento. El sitio I2, a pesar de lo mostrado por el BMWP, tiene indicios de poseer buena calidad del agua a pesar de su ubicación a pocos metros de I1. Estos indicadores serían la presencia de la sub-familia Tanypodinae y el género Psephenops, asociados a buena calidad del agua (Chalar et al., 2011; Molineri et al., 2020). Psephenops, junto con toda la familia Psephenidae, son descritos para el Uruguay como taxa muy sensibles a la contaminación (Challar et al., 2011; Arocena et al., 2018), sin embargo, Junqueira et al. (2000) halló esta familia en ambientes ligeramente contaminados (BMWP- Brasil = 63).

La correlación negativa de la zona de Recuperación con los dos primeros factores del ACP indicaría que se trata de un ambiente con peor calidad del agua que Control y que presenta ambientes arenosos-fangosos, como lo muestran las mayores abundancias de Nais sp. Müller, 1773 (Gaviria, 1993); indicando que aún no se ha logrado una completa recuperación del sistema. Varios indicadores no dieron diferencias entre Recuperación y Control o Impacto, como \%Chiro, \%spp.tol, Baetidae y Palaemonidae, lo que sugiere un proceso de mejora solo parcial de las aguas. Por otra 
parte, los géneros Hagenulopsis y Aegla que solo se observaron en $\mathrm{R}$, han sido señalados como indicadores de buena calidad de agua (Metcalfe, 1994; Figueroa et al., 2003; Arocena et al., 2018). A pesar de no se observarse diferencias significativas, se observan cambios en los índices bióticos, abundancias de especies indicadoras y parámetros comunitarios que indicarían cierta mejora en la calidad ambiental.

En este estudio se evidencia el impacto de la ciudad en las comunidades de macroinvertebrados como las familias Palaemonidae y Baetidae y el género Tricorythodes. Contrariamente, el orden Acari fue indicativo de mala calidad del agua, Ambrioso (2014), en un estudio del Río de la Plata y sus afluentes, le asignó a Acari un nivel de tolerancia igual a Chironomidae, lo cual es consistente con todos los resultados obtenidos en este estudio.

\section{CONCLUSIONES}

En el tramo de río estudiado no se observa una recuperación total debido a que todos los indicadores indican peor calidad en Recuperación que en Control. Frente a esta situación se debe buscar sitios aún más aguas abajo para determinar si se observa este patrón de mejora. Por otro lado, se observa una caída abrupta en la calidad del agua inmediatamente después de la ciudad.

Se logró diferenciar tres zonas dentro del tramo estudiado. Se demostró el impacto que genera la ciudad sobre el río, debido a que en la zona Control posee mejor calidad de agua según los índices bióticos (\%Chiro y \%spp. tol) y la abundancia relativa de las familias Baetidae y Palaemonidae (Roldán \& Restrepo, 2008; Ambrioso, 2014).

Dentro de los indicadores utilizados el \%Chiro y \%spp. tol fueron los que demostraron mayor capacidad de distinción de nivel de contaminación. Se debe seguir estudiando algunos grupos de artrópodos como Acari, Chironomidae y Palaemonidae, cuya mayor definición taxonómica podría brindar información más precisa y facilitar el análisis de los datos.

El uso de bioindicadores es especialmente útil en grandes ríos, donde los altos caudales pueden diluir rápidamente las contaminaciones de fuentes puntuales, las que no son detectadas por métodos químicos pero que son capaces de afectar significativamente a la fauna presente, permitiendo determinar efectos en el ambiente, aunque la fuente del disturbio no se encuentre en el mismo o no capaz de ser medido en el monitoreo tradicional.

\section{BIBLIOGRAFÍA}

Ambrosio E.S. 2014. Hábitats y grupos funcionales alimentarios de los macroinvertebrados bentónicos en la costa bonaerense del río de la plata: relación con la contaminación. Tesis de Doctorado, Facultad de Ciencias Naturales y Museo - UNLP.

Amestoy Rosso F.J. 2001. Hacia una cuantificación de estrés ecológico en el embalse de Rincón del Bonete (Uruguay). Tesis de Doctorado en Biología, PEDECIBA, Facultad de Ciencias, Universidad de la República, Uruguay.

Arocena R. 1996. La comunidad bentónica como indicadora de zonas de degradación y recuperación en el arroyo Toledo (Uruguay). Revista de Biología Tropical, 44(2 A): 659-671.

Arocena R. \& D. Conde. 1999. Métodos en Ecología de Aguas Continentales, con Ejemplos de Limnología en Uruguay. DIRAC/FC/UDELAR, Montevideo, Uruguay.

Arocena R., Chalar G., Perdomo C., Fabián D., Pacheco J.P., González M., Oliveiro V., Silva M. \& P. García. 2011. Impacto de la producción lechera en la calidad del agua. Serie FPTA №60 INIA: 18-28.

Arocena R., Chalar G., González I., García P., Piccardo A., Piccini C., Vernassa T., Sandes P. \& M. Castro. 2018. Evaluación ecológica de cursos de agua y biomonitoreo cuenca del Rio Negro. Informe final del Convenio de entre MVOTMA (DINAMA) y Facultad de Ciencias (UdelaR). www. limno.fcien.edu.uy

Baptista D.F., Buss D.F., Egler M., Giovanelli A., Silveira M.P. \& J.L. Nessimian. 2007. A multimetric index based on benthic macroinvertebrates for evaluation of Atlantic Forest streams at Rio de Janeiro State, Brazil. Hydrobiologia, 575(1): 83.

Blocksom K.A. \& B. R. Johnson 2009. Development of a regional macroinvertebrate index for large river bioassessment. Ecological indicators, 9(2): 313-328.

Brinkhurst R. \& M. Marchese. 1992. Guía para la identificación de oligoquetos acuáticos continentales de Sud y Centro América. Colección Climax № 6, Segunda edición. Asoc. Cs. Nat. del Litoral,Santa Fe, Argentina.

Brugnoli E., Dabezies M.J., Clemente J.M. \& P. Muniz. 2011. Limnoperna fortunei (Dunker 1857) en el sistema de embalses del Rio Negro, Uruguay. Oecologia Australis, 15(3): 576-592.

César I.I., Martín S.M. \& M.F. Colla. 2019. The Use of Littoral Benthic Macroinvertebrates of the Martín Garcia Island Nature Reserve as Indicators of Water Quality. Annu. Res. Rev. Biol., 1-22.

Chalar G., Arocena R., Pacheco J.P. \& D. Fabián. 2011. Trophic assessment of streams in Uruguay: A Trophic State Index for Benthic Invertebrates (TSI-BI). Ecol. Indic., 11: 362-369.

Conde D., Paradiso M., Gorga J., Brugnoli E., De león L. \& M. Mandiá. 2002. Problemática de la calidad de agua en el sistema de grandes embalses del Río Negro (Uruguay). Revista CIER, 39: 51-68.

DINAMA. 2011. Monitoreo y evaluación de calidad de 
agua. Plan para la definición de una línea de base del Río Negro. 2011. Informe Técnico. Departamento de Calidad Ambiental. División Evaluación de la Calidad Ambiental. 35 p. http:/ /www.mvotma.gub.uy

Fernández H. \& E. Domínguez. 2001. Guía para la determinación de los artrópodos bentónicos sudamericano. Ed. Univ. de Tucumán. Tucumán, Argentina.

Figueroa R., Valdovinos C., Araya E. \& O. Parra. 2003. Macroinvertebrados bentónicos como indicadores de calidad de agua de ríos del sur de Chile. Rev. Chil. Hist. Nat., 76: 275-285.

Flint K.R. \& A.P. Davis. 2007. Pollutant Mass Flushing Characterization of Highway Stormwater Runoff from an Ultra-Urban Area. J. Environ. Eng., 133: 616-626.

Gaviria E.A. 1993. Claves para las especies colombianas de las familias Naididae y Tubificidae (Oligochaeta, Annelida). Caldasia, 237-248.

González-Piana M., Fabián D., Piccardo A. \& G. Chalar. 2017. Dynamics of total microcystin LR concentration in three subtropical hydroelectric generation reservoirs in Uruguay, South America. Bulletin of environmental contamination and toxicology, 99(4): 488-492.

Hammer Ø., Harper D.A. \& P.D. Ryan. 2001. PAST: Paleontological statistics software package for education and data analysis. Palaeontologia electronica, 4: 1-9.

Hellawell J.M. 1986. Biological Indicators of Freshwater Pollution and Environmental Management. Elsevier Applied Science Publishers, London.

Junqueira M.V., Amarante M.C., Dias C.F.S. \& E.S. França. 2000. Biomonitoramento da qualidade das águas da Bacia do Alto Rio das Velhas (MG/Brasil) a través de macroinvertebrados. Acta Limnologica Brasiliensia, 12(1): 73-87.

Lee J.H. \& K.W. Bang. 2000. Characterization of urban stormwater runoff. Water research, 34(6): 17731780.

Lopretto C.E. \& G. Tell. 1995. Ecosistemas de aguas continentales. Metodologías para su estudio. Ed. Sur, Tomo I, II y III. La Plata, Argentina.

Merrit R.W. \& K.W. Cummins. 1996. An introduction to the Aquatic insects of North America. Hunt Pub. Co. Iowa, USA.

Metcalfe Smith J.L. 1994. Biological waterquality assessment of rivers: use of macroinvertebrate communities. The rivers handbook: hydrological and ecological principles: 144-170.

Molineri C., Tejerina E.G., Torrejón S.E., Pero E.J.I. \& G.E. Hankel. 2020. Indicative value of different taxonomic levels of Chironomidae for assessing the water quality. Ecol. Indic., 108.

Montes R.T., Navarro I., Domínguez R. \& B. Jiménez. 2013. Modificación de la capacidad de autodepuración del río Magdalena ante el cambio climático. Tecnología y ciencias del agua, 4(5): 71-83.

Moog O. 1993. Quantification of daily peak hydropower effects on aquatic fauna and management to minimize environmental impacts. Regul. Rivers: Res. Manage, 8:5-14.

Morais S., Molozzi J., Viana A., Viana T. \& M. Callisto. 2010. Diversity of larvae of littoral Chironomidae (Diptera: Insecta) and their role as bioindicators in urban reservoirs of different trophic levels. Brazilian J. Biol., 70: 995-1004.

Munn M.D. \& M.A. Brusven. 1991. Benthic macroinvertebrate communities in nonregulated and regulated waters of the clearwater river, Idaho, U.S.A. Regulated Rivers Research \& Management, 6: 1-11.

Murphy J.F., Jones J.I., Pretty J.L., Duerdoth C.P., Hawczak A., Arnold A. \& D. Hornby. 2015. Development of a biotic index using stream macroinvertebrates to assess stress from deposited fine sediment. Freshwater Biology, 60(10): 2019-2036.

MVOTMA-DINAMA. 2009. Informe de calidad ambiental de la cuenca del Río Negro https:// www.mvotma.gub.uy

MVOTMA-DINAMA. 2011. Monitoreo y evaluación de calidad de agua. Plan para la Definición de una Línea de Base para el Río Negro División Evaluación de la Calidad Ambiental. Informe de Consultoría (Proyecto PNUD-URU/07/01)

MVOTMA-DINAMA. 2017. Informe de calidad ambiental de la cuenca del Río Negro 2009 2017 https://www.dinama.gub.uy

Parra O. \& J. Rojas. 2003. Conceptos básicos sobre medioambiente y desarrollo sustentable. INETGTZ, Buenos Aires, Argentina.

Pave P.J. \& M. Marchese. 2005. Invertebrados bentónicos como indicadores de calidad del agua en ríos urbanos (Paraná-Entre Ríos, Argentina). Ecología austral, 15(2): 183-197.

Plafkin J.L., Barbour M.T., Porter K.D., Gross S.K. \& R. M. Hughes. 1989. Rapid assessment protocols for use in streams and rivers: benthic macroinvertebrates and fish. EPA/444/4-89-001, Office of Water, US EPA, Washington DC, USA.

Reavie E.D., Jicha T.M., Angradi T.R., Bolgrien D.W. \& B.H. Hill. 2010. Algal assemblages for large river monitoring: Comparison among biovolume, absolute and relative abundance metrics. Ecological Indicators, 10: 167-177.

Reynoldson T.B. 1987. The role of environmental factors in the ecology of tubificid oligochaetesan experimental study. Ecography, 10(4): 241-248.

Roldán G. \& J.J.R. Restrepo. 2008. Fundamentos de Limnología Neotropical (Vol. 15). Universidad de Antioquia, Medellin, Colombia.

Rosenberg D.M. \& V.H. Resh. 1993. Freshwater biomonitoring and benthic acroinvertebrates. 
Chapman \& Hall, New York, New York, USA.

Sabater S., Donato J.C., Giorgi A. \& A. Elosegui. 2009. El río como ecosistema. En: Elosegi A., Sabater S. \& Cortés I. (Eds.) Conceptos y técnicas en ecología fluvial, pp. 23-38. Fundación BBVA, Velant, España.

Salcedo S., Artica L. \& F. Trama. 2013. Benthic macro invertebrates as water quality indicators in the San Alberto micro basin, Oxapampa, Perú. Apuntes de Ciencia \& Sociedad, 3(2): 124-139.

Sánchez M. 2005. El índice biológico BMWP (Biological Monitoring Working Party Score), modificado y adaptado al cauce principal del Río Pamplonita Norte de Santander, Bistua, 3(2): 54-67.

Trivinho-Strixino S. \& G. Strixino. 1995. Larvas de Chironomidae do Estado de São Paulo: guia de identificação e diagnose dos gêneros. UFSCar, PPG-ERN, São Carlos, Brasil.

Valdés-Abellán J., Candela L., Jiménez-Martínez J. \& J.M. Saval-Pérez. 2013. Brackish groundwater desalination by reverse osmosis in southeastern Spain. Presence of emerging contaminants and potential impacts on soil-aquifer media.
Desalination and Water Treatment, 51(10-12): 2431-2444.

Valdez E. \& A. Vázquez. 2003. Ingeniería de los sistemas de tratamiento y disposición de aguas residuales. Fundación ICA, AC México, DF, México.

Vergara-Olaya D.L. 2009. Entomofauna lótica bioindicadora de la calidad del agua (Tesis de Grado en Medio Ambiente y Desarrollo). Facultad de Minas, Universidad Nacional de Colombia, Medellín, Colombia.

Wilhm J.L. \& T.C. Dorris. 1968. Biological Parameters for Water Quality Criteria. Bioscience, 18, 477481.

Zilli F. \& A.M. Gagneten. 2005. Efectos de la contaminación por metales pesados sobre la comunidad bentónica de la cuenca del arroyo Cululú (río Salado del Norte, Argentina). Interciencia, 30(3): 159-165.

Fecha de Recepción: 16 de septiembre de 2020 Fecha de Aceptación: 01 de diciembre 2020 